\title{
BMJ Open Qualitative study to explore radiologist and radiologic technologist perceptions of outcomes patients experience during imaging in the USA
}

\author{
Monica Zigman Suchsland (D , , Maria Jessica Cruz, ${ }^{2}$ Victoria Hardy, ${ }^{3}$ \\ Jeffrey Jarvik, ${ }^{4}$ Gianna McMillan, ${ }^{5}$ Anne Brittain, ${ }^{6}$ Matthew Thompson ${ }^{1}$
}

To cite: Zigman Suchsland M, Cruz MJ, Hardy V, et al. Qualitative study to explore radiologist and radiologic technologist perceptions of outcomes patients experience during imaging in the USA. BMJ Open 2020;10:e033961. doi:10.1136/ bmjopen-2019-033961

- Prepublication history for this paper is available online. To view these files, please visit the journal online (http://dx.doi. org/10.1136/bmjopen-2019033961).

Received 30 August 2019 Revised 25 February 2020 Accepted 02 June 2020
Check for updates

(C) Author(s) (or their employer(s)) 2020. Re-use permitted under CC BY-NC. No commercial re-use. See rights and permissions. Published by BMJ.

For numbered affiliations see end of article.

Correspondence to Monica Zigman Suchsland; mzigman@uw.edu

\section{ABSTRACT}

Objective We aimed to explore the patient-centred outcomes (PCOs) radiologists and radiologic technologists perceive to be important to patients undergoing imaging procedures.

Design We conducted a qualitative study of individual semi-structured interviews.

Participants We recruited multiple types of radiologists including general, musculoskeletal neuroradiology, body and breast imagers as well as X-ray, ultrasound, CT or MRI radiologic technologists from Washington and Idaho.

Outcome Thematic analysis was conducted to identify themes and subthemes related to PCOs of imaging procedures.

Results Ten radiologists and six radiology technologists participated. Four main domains of PCOs were identified: emotions, physical factors, knowledge and patient burden. In addition to these outcomes, we also identified patient and provider factors that can potentially moderate these outcomes.

Conclusions Radiologists and technologists perceived outcomes related to the effect of imaging procedures on patients' emotions, physical well-being, knowledge and burden from financial and opportunity costs to be important to patients undergoing imaging procedures. There are opportunities for the radiology community to measure and use these PCOs in comparisons of imaging procedures and potentially identify areas where these outcomes can be leveraged to drive a more patientcentred approach to radiology.

\section{INTRODUCTION}

The primary focus of imaging test evaluation involves establishing evidence of diagnostic accuracy. ${ }^{1}$ There is, however, a growing interest in looking beyond accuracy for additional metrics to more fully evaluate the outcomes of imaging procedures. ${ }^{2} 3$ Broadening the scope of how imaging tests are evaluated may lead to more nuanced understanding of the impact tests might have on patient outcomes. Numerous frameworks have been developed to guide the evaluation of imaging tests; ${ }^{4-8}$ one of the earliest,

\section{Strengths and limitations of this study}

- Semi-structured interviews from radiologists and radiologic technologists is an appropriate method to identify outcomes that radiologists and radiologic technologists perceive as important to patients during imaging.

- Thematic analysis was used to identify thematic patient outcomes and experiences radiologists and radiology technologists reported in their patient populations.

- We believe this is one of the first studies to include the perspectives of radiologic technologists in the exploration of patient-centred outcomes of imaging tests.

- A potential weakness of the study is that interviewing both radiologists and technologists covering a variety of imaging specialities and healthcare settings provides some generalisability of perspectives, but may not generalise to all imaging modalities, geographical regions or practice settings.

- A potential weakness is that patient outcomes reported in this manuscript were not obtained from the patient perspective, but may still be of importance.

by Fineberg, introduced a hierarchical framework that includes patient outcomes. ${ }^{9}$ Indeed, this framework placed patient outcomes as one of the most significant measures of clinical efficacy, and suggested that evaluation should include psychological factors as well as more traditional clinical benchmarks. ${ }^{9}$ Other researchers have expanded on this model, but all include patient outcomes at or near the top of evidence in effectiveness research. ${ }^{4-8}$

Patient outcomes were a somewhat nebulous concept in the original Fineberg framework. Since then, patient-centred outcomes (PCOs) have been defined as: 'an assessment of harms and benefits highlighting comparisons and outcomes that matter to people; a focus on outcomes that people notice and 
care about; and the incorporation of a wide variety of settings and diversity of participants' and have emerged as a research priority. ${ }^{10}$ Domains of PCOs can include: emotional (psychological), social, cognitive, behavioural, physical and cost. ${ }^{112}$ Although evidence to support these domains and outcomes specific to imaging testing has been limited, research from patients to date has identified PCOs related to impacts on emotions, the value of the information gained, as well as physical side effects from the testing process. ${ }^{13}{ }^{14}$ However, there has been little research exploring what radiologists and their care teams, specifically radiologic technologists, perceive as most important to patients. With growing calls for the radiology profession to embrace a more patient-centred approach, understanding the insights of the entire care team may provide key enlightenment into PCOs. Technologists are frontline staff and as a result have direct contact with patients that is unique and this provides them with perspectives that other providers do not have. We aimed to identify the outcomes that radiologists and radiologic technologists perceive as important to patients during imaging.

\section{METHODS}

We conducted a qualitative study using individual semistructured interviews with radiologists and radiologic technologists, as part of a mixed methods research programme called Patient Centered Outcomes of Diagnostics (PROD), which aims to develop new methods to guide research and comparison of imaging procedures.

Participants were recruited using a convenience sample from sites within a 5-state state (Washington, Wyoming, Alaska, Montana and Idaho (WWAMI)) practice-based research network, the WWAMI Practice and Research Network, as well as contacts through a radiologist on our research team. Volunteers were solicited through email. Participants were eligible if they were either an X-ray, ultrasound, CT or MRI technologist or general, musculoskeletal, neuroradiology, body or breast radiologist. Interested participants provided oral consent to be interviewed and were compensated with a gift card for participation.

The interview guides developed by the study team (MZS, $\mathrm{JGJ}, \mathrm{AB}$ and $\mathrm{MJT}$ ) and were informed by the previous diagnostic evaluation frameworks and PCOs reported from previous research with patients, as well as feedback from the PROD study Stakeholder Advisory Board (consisting of patients, clinicians, researchers, industry and scientific organisations). ${ }^{11} 1315$ Interview questions were framed to follow the testing timeline of before, during and after imaging testing. The interview guides included a brief introduction about the study goals and questions on participant demographics, then remaining questions focussed on interviewees' roles in caring for patients, determining test appropriateness, communicating with patients and observations of patient experiences. Both a radiologist and a radiologic technologist on the research team reviewed each interview guide for relevance and appropriateness to the job roles.

Data collection occurred from February 2017 to December 2017. Enrolled subjects participated in a single semi-structured interview in person or by phone. Interviews lasted from 45 to $60 \mathrm{~min}$ and were conducted by a trained interviewer (MZS). The interviewer did not have a prior relationship with study participants and was identified as a research coordinator to the participants. Interviews were audio recorded and transcriptions were checked for accuracy. Interviews $(n=16)$ were conducted until data saturation was achieved, defined as: no additional themes emerged from the interview. ${ }^{16}$

Transcripts were uploaded to qualitative analysis software (Dedoose V.7.0.23, Los Angeles, California: SocioCultural Research Consultants, LLC, www.dedoose.com). Researchers (MJC, MZS and VH) immersed themselves in the same three transcript texts. Each researcher identified text excerpts important to the research question and derived codes from this text. The researchers compared codes and refined codes as needed (MJC, MZS and VH), to develop a codebook of a defined set of codes. This codebook was applied to an additional transcript and refined through an iterative process until a final codebook was developed. Final codes included: test appropriateness, communication, interaction with the patient, outcomes from accuracy, barriers or facilitators to testing, impacts on care delivery, accommodations made for patients, perception of patient outcomes and what drives patients. Two researchers (MJC and MZS) applied the final codebook to the remaining transcripts; discrepancies in coding were reconciled by a third researcher (VH). The initial four transcripts were also reviewed using the final list of codes. Code excerpts were then reviewed by three researchers (MJC, MZS and VH) and analysed for common themes and subthemes through thematic analysis. ${ }^{17}$ Themes were compared with previous findings from the PROD study to confirm outcomes and to present novel outcomes that emerged from this new perspective. ${ }^{13} 18$

\section{Patient and public involvement}

The PROD study recruited 26 stakeholders to participate on the PROD study Stakeholder Advisory Board. There were eight patients/patient advocates, four primary care clinicians, one radiology technologist (one radiologist on the core research team), five researchers with expertise in methods evaluating diagnostic tests, four imaging industry representatives, three senior staff from the American College of Radiology and one stakeholder from a healthcare non-profit organisation. Stakeholders were involved in study design through development of the interview guide, evidence interpretation on identification of study themes and development of the manuscript. Stakeholders were not involved in recruitment or data collection for this study. Lastly, there is no formal plan to disseminate results to specific participants, we will however, share study results back to the study sites that participated as recruitment sites. 
Table 1 Characteristics of radiologists and radiologic technologists interviewed

\begin{tabular}{ll}
\hline & $\mathbf{N}=\mathbf{1 6}$ \\
\hline Gender & 6 \\
\hline Female & 10 \\
Male & \\
Race and ethnicity & 3 \\
\hline Asian & 0 \\
\hline Black or African-American & 0 \\
\hline Hispanic or Latino & 1 \\
\hline Mixed race & 12 \\
\hline White & \\
\hline Provider type & 6 \\
\hline Radiologic technologist & 10 \\
\hline Radiologist & $1975-2017$ \\
\hline Year completed training & \\
\hline Range in year & 44 (26-67) \\
\hline Age & \\
\hline Mean years (range) & 6 \\
\hline Clinical practice setting & \\
\hline Outpatient & \\
\hline Inpatient and outpatient & \\
\hline
\end{tabular}

Data availability

No additional data available.

\section{RESULTS}

Participants included 10 radiologists specialising in body (abdominal, cardiovascular, cardiothoracic), neuroradiology, musculoskeletal, generalist and breast imaging, as well as six radiologic technologists with specialities of sonography, X-ray, MRI and CT (table 1). No participants dropped out of the study due to the sampling method and short time frame.

Four domains of PCOs were identified through thematic analysis. Included in these domains are specific outcomes, as well as moderators that appear to influence these outcomes. We applied the definition of moderator as a variable that specifies when certain effects hold, such as the direction or the strength of a relationship between the predictor (in this case imaging testing) and the PCO. ${ }^{19}$ (figure 1).

\section{Emotional outcomes}

Radiology providers identified a range of emotional outcomes tied to their perceptions of patients' responses to imaging testing. Negative emotions appeared in the pre-testing phase in the form of fear, worry, stress or anxiety about future test results. Radiology providers observed that these emotions often continued through

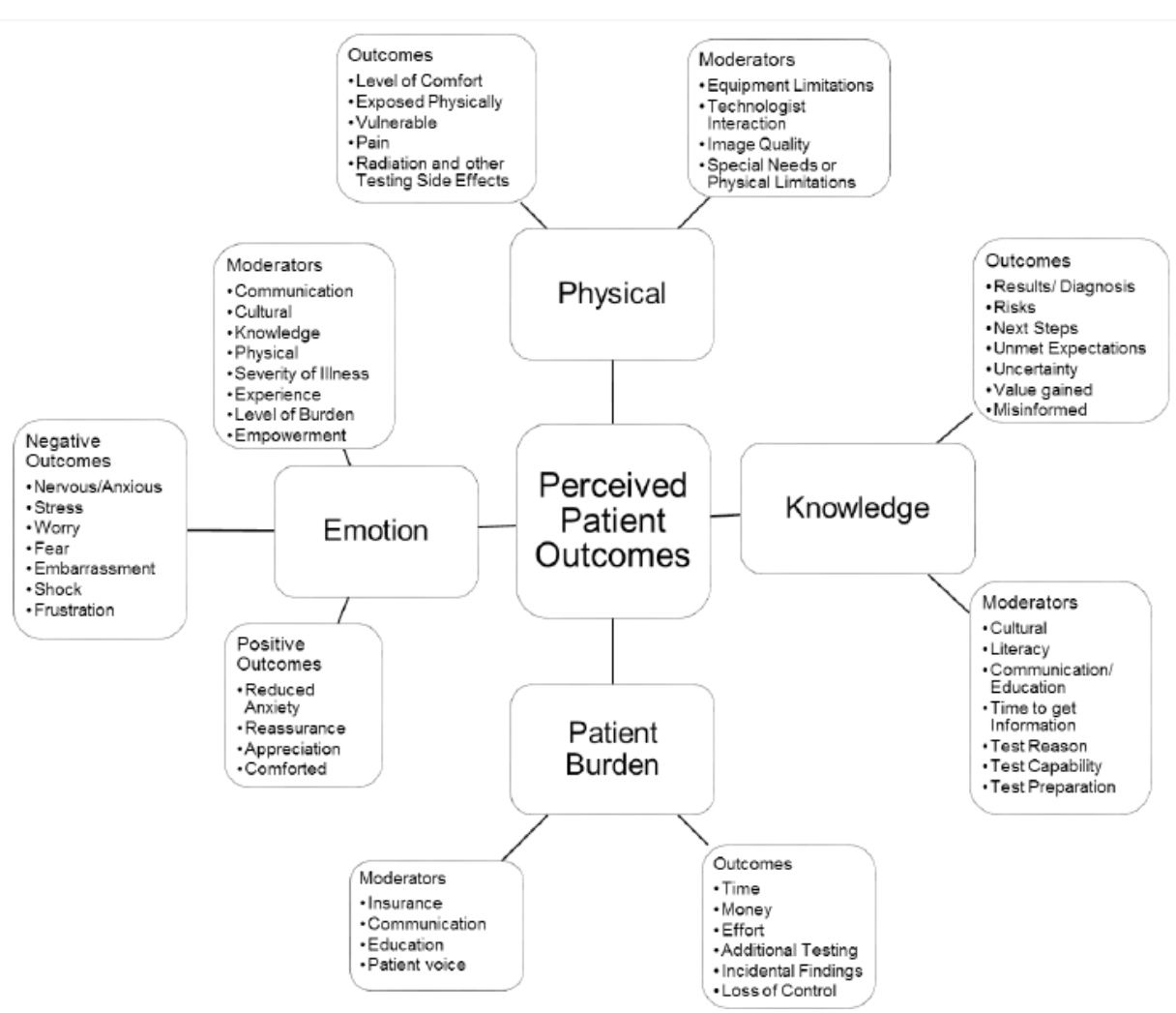

Figure 1 Domains of patient-centred outcomes from imaging procedures and potential moderating factors. Domains: physical, knowledge, patient burden and emotion can be found in the four boxes at the centre of the figure with branching boxes for specific patient outcomes and moderators that were identified for each domain. 
the process of image acquisition, described by the following radiologist:

In terms of the patient experience, people are anxious. Especially since we only do targeted ultrasound if there is a specific area of concern. When we're doing an ultrasound, they are worried about what we might be seeing. If you don't say anything, they get worried. If you take pictures, they get worried. - R9

As evidenced from the quote above, factors such as patient's level of knowledge, the physical experience and provider actions were believed to moderate patients' emotional responses to imaging. Communication often helped to attenuate negative emotions by reducing anxiety, helping patients feel comforted and reassured:

I did a breast biopsy earlier today. That woman was scared to death. She had never had one... She was worried it was gonna hurt, and I had to take more time than normal to explain things... Her impact, at least coming in, was she was very scared and nervous. When she left she was happy, because she got good care. - R8

Technologists appeared to play a particularly prominent role during the testing process in helping to induce positive emotions, as explained by one technologist:

They come in... and they're very scared... I feel like if you give them excellent patient care, I feel like it calms them down a little bit, make them feel comfortable, just reassuring them that they're in good hands, that I'll take care of them. - T4

Other factors that were identified as moderating patient's emotional reactions included patients' cultural background, the reason for testing and prior experience with imaging. A further moderator appeared to be patients' apparent loss of control over the imaging testing process, which could further influence their emotional responses.

It seems like a lot of the time that they [patients] just kind of go with the flow... It seems like a lot of them don't understand that they have the opportunity to refuse that [imaging test] or not necessarily refuse but to question exactly why they're going to be having a certain study. - T2

\section{Physical outcomes}

Patients' physical experiences were readily apparent to radiology providers, and included level of comfort or discomfort/pain, vulnerability/exposure and side effects of the testing procedure. A number of technologists described that making the patient as comfortable as possible sometimes conflicted with their goal of trying to obtain optimal quality of images. This was sometimes complicated by the particular needs of a patient and the restrictions of imaging modalities to meet these needs (eg, patient body size or physical limitations). One technologist explained the interactions between physical discomfort and image quality:

She was just hurting, it just hurt. And I tried to make sure that I didn't make it worse. So she cried the whole time... You just kind of have to do it [imaging test], and try to be careful. - T1

Interviewees felt that some patients are particularly vulnerable or physically exposed during imaging. This physical experience was often perceived as being influenced by patients' cultural backgrounds:

There's a certain population around our area and just people in general depending on how you were raised, that disrobing is completely like, whoa, you just asked me to take my clothes off. That's something that I've come across a few times, but people are like, well, I'm not so sure I can do that. - T2

Radiology providers were aware of physical adverse reactions to contrast media and radiation exposure. While acknowledging these risks, their level of communication about them to patients varied among providers, but was typically minimised.

I think there's almost no risk to any diagnostic test we do... Apart from bad hardware interactions with MRI... There's a baseline risk to using ionising radiation, but it's tremendously low. It's one of those things that you have to validate the concern, but at the same time trying to explain that there's no real concern... I think it's because it's a lack of familiarity on the patient's part of how the stuff works and people worry about stuff. - R1

\section{Knowledge outcomes}

Radiology providers noted several outcomes within the domain of knowledge. One outcome was the extent to which the imaging test was able to fulfil patients' expectations of the information they hoped to gain from the test. An expectation that testing would yield answers to a patient's concerns, and pressure from the patient themselves to conduct imaging to 'find the answer' was noted by interviewees:

I think that imaging is kind of a necessity in the patient's mind now. It used to be prescription drugs, but it's like they come into the emergency department or their doctor's office and they expect us to look inside their body somehow and give them an answer. I think it's just a huge role for the patient's peace of mind even. Like I've got chest pain, I want you to do a chest $\mathrm{X}$-ray... - T6

At other times, expectations to provide answers could not be met. This occurred with patients who appeared to have unrealistic expectation of the imaging procedure.

A lot of times there's unreal expectations placed on an exam where they think they're going to be getting 
an answer. It's just not realistic to expect a diagnosis of the test typically. We're not pathologists. We don't see the actual cells. - R3

Radiologists acknowledged that patients are often uninformed about why they were getting the test, what they should expect and what the information would lead to:

I feel like they're [patients] probably mostly in the dark. A lot of times they're not really sure why they're getting the exam. They are never aware of what's actually going to be seen on the exam. I would say the whole process is kind of hidden from the patient. I'm sure they would appreciate being more informed on what is going on and why... - R3

A further outcome within the domain of knowledge focussed on how and what to inform patients including communication about test results, next steps in their healthcare, risks of testing and test indications.

I found the more information you give people about what is actually going on, the more receptive they are and relaxed about it. People are really afraid of the unknown, and there's a ton of unknowns in MRI. - T5

While providing clear communication was seen as positive by some providers, others struggled to know how much to share with a patient, and was sometimes moderated by challenges with language, literacy level and cultural differences.

Also, there is a risk of telling people too much. When they don't want to have life-saving procedures because they're freaked out. You don't want to tell them what to do or manipulate them, but there's a line. You can also set expectations. If you tell them, 'This is going to be unpleasant and cold,' ... Again, you don't want to be dishonest, and you want to be honest, but you also don't want to suggest things that they may not experience. $-\mathrm{R} 6$

\section{Patient burden}

Radiology providers recognised several burdens related to the imaging process that they considered important outcomes for patients. First, was the time and opportunity costs of having the imaging test, such as time off work, travel times particularly for patients living in rural areas or waiting time to get the test performed. Another outcome was the financial burden and the extent to which insurance would or would not cover the costs of the imaging:

There's all sorts of roadblocks. Especially an outpatient test, because you have to be off that day or get time off from work, go to this outpatient centre, wait in a waiting room. It takes a lot of time. Another obstacle is having insurance. If you don't have insurance, then getting a really expensive test is difficult. You might even have to pay for it yourself.-R7
At times, these burdens were seen as barriers to obtaining needed tests. Burden was moderated by insurance denying coverage, how the patient was informed (communication and education) or the level of importance placed on the patient voice (patient concerns) and how that was addressed. Outcomes in this domain include out-of-pocket money, time (to schedule, take the test, get the results, to get answers to questions or concerns), amount of imaging or effort (due to incidental findings or wrong image taken).

\section{DISCUSSION \\ Main findings}

Radiologists and radiologic technologists describe multiple different outcomes that they perceive as important for their patients undergoing imaging testing. These included outcomes related to patients' emotional reactions (eg, reassurance or anxiety), outcomes from the physical effects of testing (eg, discomfort and test side effects), those related to the information gained (eg, to help explain symptoms or to answer patient's questions) and outcomes related to the burden of the procedure (eg, financial and opportunity costs). These outcomes did not occur in isolation from the care team, but were often strongly influenced or moderated by the radiologist or radiologic technologist. Other factors that could modify the outcomes experienced by patients included patients' previous experiences, their underlying health status, baseline level of knowledge, their self-efficacy (usually identified as loss of control within the testing environment), expectations of the imaging test (realistic and unrealistic), insurance status and cultural background.

We compared outcomes where possible from perspectives of radiologists and radiologic technologists. Apart from a minority of radiologists (those specialised in breast, interventional or overseeing contrast injections), most had minimal direct 'face to face' contact with patients. Most radiologists felt that their primary client was the clinician ordering the test, and not necessarily the patient. In contrast, radiologic technologists acted as the primary point of contact with patients during imaging, serving as the main source of communication between the ordering provider, radiologist and patient. Technologists had tremendous opportunity to address or influence patients' emotions, knowledge and some physical and patient burdens at point of care. In their interaction with patients, technologists were often able ease fears, comfort patients, meet special needs, listen to patients and set patient expectations. Indeed, a recent study on musculoskeletal imaging found that staff had an overall positive impact on patient experience of testing. ${ }^{20}$ A second study confirms the importance of technologists over radiologists and their behaviour in patients' valuation of excellent care. ${ }^{21}$ Technologists' inability to provide imaging test results (as this is outside their scope of practice), sometimes led to patient frustration and anxiety for those wanting immediate answers. 


\section{Comparison with existing literature}

Several of the PCOs characterised by this study have been previously identified by the research team during two previous studies, one from the perspectives of patients themselves and one from the perspectives of referring clinicians. Patients identified knowledge gained, contributions to healthcare, experiences during testing and impacts on emotion as patient-centred outcomes of imaging tests. ${ }^{13}$ Primary care providers were able to connect the outcome pathways a bit more and reported that the answers provided from imaging tests influenced emotional outcomes and that there is additional burden on the patient from added testing, monetary and physical risks. ${ }^{18}$ This evidence of triangulation from the four perspectives strengthens support for the occurrence and potential importance of these outcomes among patients undergoing imaging testing. ${ }^{13}$ Indeed, previous research has highlighted the importance of psychosocial/emotional outcomes (often described as stress, anxiety or reassurance), ${ }^{22-24}$ as well as physical impacts of tests (including comfort/discomfort) ${ }^{25}$ and the value of information to patients such as knowledge about the test, awareness of harms, value (or lack of value) of knowing test findings. ${ }^{132025-27}$ In contrast, the burden of testing to patients has received less attention, apart from the issue of waiting times and its impact on emotions and life planning, as well as the issue of burden from loss of control that some patients experience. ${ }^{20} 25$ Other outcomes that were reported in the literature were not identified from our interviews, such as impact on behavioural or social outcomes. ${ }^{1120222526}$ It is possible that these additional outcomes might be more prominent in longer-term follow-up to imaging testing and may be important to explore in future studies.

\section{Strengths}

This study provides a novel insight into the awareness that radiology providers have about the outcomes that patients experience when undergoing imaging exams. In particular, we believe this is one of the first studies to include the perspectives of radiologic technologists who provided particular insight based on their key roles within the imaging process. Technologists' direct contact with patients provides a unique perspective radiologists cannot provide. Past research has been limited to disease or modality specific topics. We believe that covering a variety of imaging specialities and healthcare settings is a strength of this study because it can corroborate past research and has found commonality across modalities/ settings.

\section{Limitations}

While qualitative research provides a high level of depth on a topic, our findings are limited due to the small sample of providers whose experiences may not be generalisable to other imaging modalities, geographical regions or practice settings. While data saturation was achieved, the sample size was small. A convenience sample of providers was chosen further creating sampling bias. These results should be confirmed in a wider random population of radiology providers. We also do not know the relative importance of outcomes to patients (and providers); these should be evaluated through additional qualitative research and validated through quantitative methods. The researchers also recognise that their thematic analysis may have been influenced by previous research that they have conducted in this area or biassed from their own perspectives.

\section{Implications for research, clinical care, patients}

What do our findings mean for the radiology profession? It is clear that the full value of imaging testing involves more than 'just' providing an accurate and timely test result. There seem to be a complex array of outcomes related to patients' emotional, physical factors and patient burden domains that occur during imaging, on top of the perceived and actual value of the information provided by the test. This implies that comparing tests solely based on their comparative accuracy may be insufficient, and risks can both be underestimated or overestimated in the benefit/risk equation of imaging procedures. At present however, these outcomes are not routinely measured or reported in current comparative studies of diagnostic tests nor do we know how to rank or prioritise them within a patient's overall experience and outcomes. ${ }^{14}$ If the field of radiology moves to change direction to become more of a patient-centric speciality, it will be necessary to find tools to measure these outcomes, prioritise (or weight) these outcomes and devise ways to incorporate them within shared decision-making with patients.

\section{Author affiliations}

${ }^{1}$ Department of Family Medicine, University of Washington, Seattle, Washington, USA

${ }^{2}$ Department of Psychiatry and Behavioral Sciences, Stanford University, Stanford, California, USA

${ }^{3}$ Department of Public Health and Primary Care, University of Cambridge, Cambridge, UK

${ }^{4}$ Departments of Radiology, Neurological Surgery and Health Services, and the Comparative Effectiveness, Cost and Outcomes Research Center, University of Washington, Seattle, Washington, USA

${ }^{5}$ Bioethics Institute, Loyola Marymount University, Los Angeles, California, USA ${ }^{6}$ Quality Improvement and Outcomes Department, Inova Fairfax Medical Campus, Falls Church, Virginia, USA

Acknowledgements The authors acknowledge the support and contributions of the PROD Study Team, a body of stakeholders, study site champions and coordinators, and researchers. This study was supported by the National Center for Advancing Translational Sciences of the National Institutes of Health under Award Number UL1 TR002319. The content is solely the responsibility of the authors and does not necessarily represent the official views of the National Institutes of Health.

Contributors MZS, research scientist on the project - implemented the study design; collected the data; analysed and interpreted the data; drafted and revised the work; final approval of the version to be published; agreed to be accountable. MJC, research assistant on the project - analysed and interpreted the data; revised the work; final approval of the version to be published; agreed to be accountable. $\mathrm{VH}$, research scientist on the project - analysed and interpreted the data; revised the work; final approval of the version to be published; agreed to be accountable. $\mathrm{JJ}$, key investigator expertise in radiology research - helped design the study; recruited study participant; and interpreted results; substantially revised the work for intellectual content; final approval of the version to be published; agreed to be 
accountable. GM, patient advocate stakeholder on the PROD study - interpreted results; substantially revised the work for intellectual content; final approval of the version to be published; agreed to be accountable. $A B$, radiology stakeholder on the PROD study - interpreted results; revised the work for intellectual content; final approval of the version to be published; agreed to be accountable. MT, principal investigator - designed the study, analysed and interpreted the data; substantially revised the work for intellectual content; final approval of the version to be published; agreed to be accountable.

Funding This study was supported through a Patient-Centered Outcomes Research Institute (PCORI) Program Award (ME-1503-29245) to derive new methods to incorporate patient-centered outcomes in studies of diagnostic imaging studies (the Patient-Centered Outcomes for Diagnostics, or PROD study).

Competing interests Dr JJ is a Section Editor and consultant for UpToDate; has received travel reimbursement from the General Electric-Association of University of Radiologists Radiology Research Academic Fellowship (GERRAF) for service on the faculty advisory board; is a Co-Editor of Evidenced-based Neuroradiology published by Springer. Ms MZS, Ms MJC, Ms VH, Dr GM, Dr AB and Dr MT have no conflicts of interest.

Patient consent for publication Not required.

Ethics approval This study was approved by the University of Washington Human Subjects Division.

Provenance and peer review Not commissioned; externally peer reviewed.

Data availability statement № data are available. No additional data available.

Open access This is an open access article distributed in accordance with the Creative Commons Attribution Non Commercial (CC BY-NC 4.0) license, which permits others to distribute, remix, adapt, build upon this work non-commercially, and license their derivative works on different terms, provided the original work is properly cited, appropriate credit is given, any changes made indicated, and the use is non-commercial. See: http://creativecommons.org/licenses/by-nc/4.0/.

ORCID iD

Monica Zigman Suchsland http://orcid.org/0000-0001-7007-6973

\section{REFERENCES}

1 Hollingworth W, Jarvik JG, Surgery N. Technology assessment in radiology: putting the evidence in evidence-based radiology. Radiology 2007;244:31-8.

2 Van den Bruel A, Cleemput I, Aertgeerts B, et al. The evaluation of diagnostic tests: evidence on technical and diagnostic accuracy, impact on patient outcome and cost-effectiveness is needed. J Clin Epidemiol 2007;60:1116-22.

3 Mustafa RA, Wiercioch W, Ventresca M, et al. Decision making about healthcare-related tests and diagnostic test strategies. paper 5: a qualitative study with experts suggests that test accuracy data alone is rarely sufficient for decision making. $J$ Clin Epidemiol 2017;92:47-57.

4 Fryback DG, Thornbury JR. The efficacy of diagnostic imaging. Med Decis Making 1991;11:88-94.

5 Ferrante di Ruffano L, Hyde CJ, McCaffery KJ, et al. Assessing the value of diagnostic tests: a framework for designing and evaluating trials. BMJ 2012;344:e686.

6 Gazelle GS, Kessler L, Lee DW, et al. A framework for assessing the value of diagnostic imaging in the era of comparative effectiveness research. Radiology 2011;261:692-8.
7 Swan JS, Pandharipande PV, Salazar GM. Developing a patient-centered radiology process model. J Am Coll Radiol 2016;13:510-6.

8 Lijmer JG, Leeflang M, Bossuyt PMM. Proposals for a phased evaluation of medical tests. Med Decis Making 2009;29:E13-21.

9 Fineberg HV, Bauman R, Sosman M. Computerized cranial tomography. Effect on diagnostic and therapeutic plans. JAMA 1977;238:224-7.

10 Carlos RC, Buist DSM, Wernli KJ, et al. Patient-centered outcomes in imaging: quantifying value. J Am Coll Radiol 2012;9:725-8.

11 Bossuyt PMM, McCaffery K. Additional patient outcomes and pathways in evaluations of testing. Med Decis Making 2009;29:E30-8.

12 Harris RP, Sheridan SL, Lewis CL, et al. The harms of screening. JAMA Intern Med 2014;174:281-5.

13 Zigman Suchsland ML, Witwer E, Truitt AR, et al. Patient-centered outcomes related to imaging testing in US primary care. J Am Coll Radiol 2019;16:156-63.

14 Thompson M, Hardy V, Suchsland MZ, et al. A secondary analysis to identify patient-centered outcomes in the American College of Radiology's appropriateness criteria. J Am Coll Radiol 2019.

15 Sabbatini AK, Merck LH, Froemming AT, et al. Optimizing patientcentered communication and multidisciplinary care coordination in emergency diagnostic imaging: a research agenda. Acad Emerg Med 2015;22:1427-34.

16 O’Reilly M, Parker N. 'Unsatisfactory saturation': a critical exploration of the notion of saturated sample sizes in qualitative research. Qualitative Research 2013;13:190-7.

17 Braun V, Clarke V. Using thematic analysis in psychology. Qual Res Psychol 2006;3:77-101.

18 Zigman Suchsland ML, Hardy V, Zhang Y, et al. Provider perspectives of patient experiences in primary care imaging. J Am Board Fam Med 2019;32:392-7.

19 Baron RM, Kenny DA. The moderator-mediator variable distinction in social psychological research: conceptual, strategic, and statistical considerations. J Pers Soc Psychol 1986;51:1173-82.

20 Bourke S, Taylor WJ, Doyle AJ, et al. The patient experience of musculoskeletal imaging tests for investigation of inflammatory arthritis: a mixed-methods study. Clin Rheumatol 2018;37:2261-8.

21 Rosenkrantz AB, Pysarenko K. The patient experience in radiology: observations from over 3,500 patient feedback reports in a single institution. J Am Coll Radiol 2016;13:1371-7.

22 Vis JY, van Zwieten MCB, Bossuyt PMM, et al. The influence of medical testing on patients' health: an overview from the gynecologists' perspective. BMC Med Inform Decis Mak 2013;13:117.

23 Slatore CG, Sullivan DR, Pappas M, et al. Patient-centered outcomes among lung cancer screening recipients with computed tomography: a systematic review. J Thorac Oncol 2014;9:927-34.

24 Miller LS, Shelby RA, Balmadrid MH, et al. Patient anxiety before and immediately after imaging-guided breast biopsy procedures: impact of radiologist-patient communication. J Am Coll Radiol 2013;10:423-31.

25 Agapova M, Bresnahan BW, Linnau KF, et al. Toward a framework for benefit-risk assessment in diagnostic imaging. Identifying scenariospecific criteria. Acad Radiol 2016:1-12.

26 Von Wagner C, Knight K, Halligan S, et al. Patient experiences of colonoscopy, barium enema and CT colonography: a qualitative study. Br J Radiol 2009;82:13-19.

27 Koney N, Roudenko A, Ro M, et al. Patients want to meet with imaging experts. J Am Coll Radiol 2016;13:465-70. 